\title{
Awareness and Perception of Drug Trials among Patients with Mental Disorders
}

\author{
Adebayo Sunday Adebisi ${ }^{(0)}$, Shehu Sale* \\ Department of Clinical Services, Federal Neuropsychiatric Hospital Kware, Sokoto, Nigeria \\ Email: *shehusale@yahoo.com
}

How to cite this paper: Adebisi, A.S. and Sale, S. (2019) Awareness and Perception of Drug Trials among Patients with Mental Disorders. Open Journal of Psychiatry, 9, 165-178.

https://doi.org/10.4236/ojpsych.2019.92013

Received: February 1, 2019

Accepted: April 22, 2019

Published: April 25, 2019

Copyright ( 2019 by author(s) and Scientific Research Publishing Inc. This work is licensed under the Creative Commons Attribution International License (CC BY 4.0).

http://creativecommons.org/licenses/by/4.0/

(c) (i) Open Access

\begin{abstract}
Mental disorders are generally characterized by abnormal thoughts, perceptions and emotions. These abnormalities may make an informed consent more difficult to obtain from psychiatric patients. The implementation of drug trials is being threatened by the decline in its overall frequency generally, especially in Psychiatry. This foretells a dearth of major breakthroughs in pharmacotherapy for psychiatric patients if they are unwilling to present themselves for drug trials. Insight into patient's awareness and perception of drug trials will enhance the planning, design and implementation of drug trials among this vulnerable subpopulation. This was a cross-sectional study of one hundred patients attending the follow-up clinic of a tertiary psychiatric hospital. A proforma questionnaire to assess awareness and perception of drug trials was administered to participants. About half (51\%) were aware of drug trials and $50 \%$ of the participants were not willing to participate in a drug trial. Sixty-five percent considered it highly risky to participate. Females were more likely to be unsure whether informed consent will be needed for drug trials. The married were more likely to be unaware of drug trials in clinical research. The unmarried were more likely to be informed about drug trials through the media. Findings are likely to have been influenced by the socio-cultural environment of this study setting. Drug trials among this population require an approach that will create an initial drug trial disorder-sensitive awareness and a design that will ensure that the consent process will take into cognizance the vulnerability of these patients; especially among females.
\end{abstract}

\section{Keywords}

Awareness, Drug Trials, Mental Disorders, Perception

\section{Introduction}

According to the World Health Organization (WHO), mental disorders are 
generally characterized by a combination of abnormal thoughts, perceptions, emotions, behaviours and relationship with others [1]. All these abnormalities may sometimes make working with someone with mental illness a difficult and an uneasy task. Furthermore, activities that will require an informed consent on the part of the mentally ill are even more difficult to engage in. Since clinical researches are studies in which people participate as either volunteers or as patient [2], finding people that are mentally ill to participate have always been difficult.

Clinical drug trials generate essential evidence for clinicians to make informed decisions for the care of their patient and discovery of novel drugs. As important as the process of drug trials may seem, its implementation is being threatened by the decline in the overall frequency of drug trials in general and in psychiatry in particular. Nierenberg noted that other fields of medicine are in the best of time as there had been a remarkable improvement in basic sciences like neuroimaging and genetics but psychiatry is in the worst of time due to the dearth of drug trials that assesses new drug interventions [3]. This foretells an unpalatable story for Psychiatry because there will be no major new breakthrough in patients' care if those that will need the care are unwilling to present themselves for clinical drug trials.

Little is known about the awareness and perception of drug trials among psychiatric patients. Successful implementation of drug trial among psychiatric patients will require an in-depth knowledge of the scope of awareness and perception of these patients. This is likely to be influenced by the socio-cultural environment of the study setting.

\section{Definition of the Research Problem}

Drug trial is one of the most potent method for evidence based medicine [4]. If science (medicine inclusive) is predicated on time tested evidences, then a decline in such evidence generating endeavours like drug trials can spell doom for the specific body of knowledge. It would not be an easy task to know the rate of drug trials in a sub-Saharan African country like Nigeria, but the best guess will be that a poor economy will affect the rate at which researches and by implication, drug trials are being done [5]. There are many factors that militate against people getting involved in drug trials in psychiatry among which are people awareness and perception of drug trials. Most patients suffering from mental illness are not receiving any form of treatment, albeit subjecting themselves for research endeavours that will improve their treatment and others' will help advance treatment modalities [6]. Knowing the level of awareness of the patients suffering from mental illness to drug trials and their attitude towards it will go a long way in helping the clinicians and researchers in making informed decisions about the best form of design and protocol to develop in a drug trial.

\section{Literature Review}

\subsection{Definition}

According to the world health organization (WHO), a clinical trial is any re- 
search study that prospectively assigns human participants or groups of humans to one or more health-related interventions to evaluate the effects on health outcomes [7]. While Clinical trial entails all types of medical interventions, drug trial is a subgroup of clinical trial that subsumes the use of drugs/medications as the form of intervention in the experimental study called clinical trial. It has been reported that less than $10 \%$ of Americans participates in research studies and less than 10\% reported knowing "a lot" about drug trials [8]. Another study among Americans also revealed that $32 \%$ were willing to participate in cancer clinical trials [9]. In developing countries where lower number of such research activities are carried out, the level of awareness is expected to be much lower.

\subsection{Goals}

The two major goals of drug trials are to learn about the efficacy and effectiveness of a drug and to learn about how safe a drug is. These goals are further typified by what the researchers want to achieve with the trials. This may thus be in form of Prevention, treatment, diagnostic trials, screening and to assess patients Quality of life [10].

\subsection{Phases}

Most researchers go with the notion that there are four phases to a drug trial (Phase 1 - 4), but a few others believe there are five stages to it (Phase 0 - 4) [11] [12] [13].

Phase 0: These are usually optional. It is the first-in-human trials of drug. It is equivalent to the usual test dose given to patients on potentially dangerous drugs. Here, a sub-therapeutic dose of the drug to be tested is given to a small group of individuals (10 - 15 subjects) and a preliminary data on the drug pharmacodynamics and pharmacokinetics are collected.

Phase 1: This is often the first in-human trials. It is a testing that takes between 20 - 80 subjects. It is to evaluate safety of the drug and determine the safe dosage range. It is also the stage at which side effects of the trial drug is being identified.

Phase 2: This stage deals with larger number of subjects (100 - 300). It also determines efficacy of drugs and evaluate some less common side effects. Since rarer adverse effects come to the fore with larger number of people, this stage helps in identifying uncommon side effects of the drugs being tested.

Phase 3: This entails testing with an even larger number of subjects (1000 3000). This is to confirm efficacy, evaluate effectiveness, monitor side effects and then compare these to some commonly used treatment. This is to ensure that information that will allow it to be used safely is collected.

Phase 4: This is the post marketing phase where continuous monitoring of the marketed drug is done. Information about the drug is collected throughout the lifetime of the drug use.

\subsection{Who Can Participate in Drug Trials?}

Generally speaking, anyone can participate in drug trials once an informed con- 
sent can be gotten. However, some special groups have more stringent criteria than others. In the US, people that are above 55 years are often excluded from drug trials because of their greater health issues and their different physiology in drug metabolism when compared to young people. Also pregnant women (fear of teratogens), children and people with unrelated medical conditions are often excluded [14].

\subsection{The Process of Drug Trial}

Investigators usually recruit subjects that have a predetermined characteristic into the drug trial over a period of time. There is usually a study protocol that stipulates all the aims, objectives and other terms of reference of the study as the guide to each investigator. Consenting subjects will be administered the drug or placebo (as the case may be) and data about the subjects' health will be collected for statistical analysis [15] [16].

\subsection{Safety Issues Why Protocol Eligible Subjects Refuse Participation}

The responsibility of safety of subjects is shared among the sponsors of the drug trials, the local investigator who implements the study, the ethical committee of the institution on whose subjects the drug is to be tested, and the regulatory agency of the country where the study is being done.

Some studies have been done on why protocol eligible study subjects refuse to participate in drug trials and they came with different arrays of reasons. According to Julie et al in their 2011 study, among the commonest reason for refusal in drug trials are the research protocol issues like long duration of study, use of invasive procedures like setting IV line, collecting blood, taking other body tissues sample etc. Subjects are also often weary of the side effect of drugs and the risk of symptoms getting worse during the drug washout period [13]. Some study schedules are also inconvenient to a large number of subjects like studies held during work hours, studies in which patients will have to travel a long distance and lack of flexibility in the participants' schedule. Some other reason given by subjects include concerns about confidentiality, some are interested only in trials that involve alternative medicine, some for religious reasons, while some also have a particular type of research they want to participate in, others can participate solely for pay [13] [17]. A 1991 study by Llewellyn-Thomas et al reported that out of the 60 people interviewed, only 25 of them said they can agree to a drug trial while the remaining 35 were on a negative affirmative. Two-third of those that refused were averse to the use of randomization [18].

Subjects' aversion to drug trials in psychiatry seems to be similar to what obtains in the general medical practice but with some peculiarities that are associated with patients that are mentally ill. March et al for instance noted that drug trials on mentally ill patients often fail to maximize clinical utility for practicing 
clinicians and thus has low clinical impact [19]. They suggested the use of straight forward clinical questions, use of a representative sample of subjects, randomization, and limited burdens on the subjects among other things as the solution to this. Tansella et al. noted that there were no published criteria for improving the quality and effectiveness of randomized control trials in psychiatry and suggested that the study subject sampled should be as closely related to the target population as possible. They also suggested the use of a culturally acceptable intervention and elimination of bias through the use of controls as the solution to bad study quality being experienced in psychiatry [20].

\subsection{Benefits}

Drug trials participation is beneficial to the participants and the society at large. It is the basis for generating essential evidence for clinicians to make informed decisions for the care of their patients [3]. I addition, it avails the participants access to treatment with experimental or study drugs that may not be available elsewhere. It gives participants access to care by teams that are familiar with the most advanced treatment available and to treatment that have been reviewed by many people including specialists and researchers. It gives participants the opportunity to learn about their illness and how to take care of it. There is often the availability of free drugs to the participants by virtue of their participation. Lastly, the satisfaction of helping others by contributing to knowledge or helping to find a possibly new treatment cannot be overemphasized [3] [10].

\section{Methodology}

Setting: The Federal Neuropsychiatric Hospital Kware, Sokoto is a tertiary health care facility located in Kware local government area of Sokoto State, Nigeria.

\subsection{Study Design}

It was a cross-sectional study involving patients managed for psychiatric disorder and attending the follow-up clinic at the hospital from August 2017 to November 2018.

\subsection{Ethical Consideration}

Ethical committee of Federal Neuropsychiatric Hospital Kware, Sokoto was sought to ensure that the work meets ethical standards and approval was granted by this committee.

\subsection{Questionnaire}

A proforma was developed containing items on sociodemogrphic factors. Questions to assess awareness and perception of drug trials was also included. This was designed to elicit the following:

1) Socio-demographic data e.g. gender, marital status, age, sex, educational 
level, occupation and residence.

2) Awareness and perception of the processes involved in a drug trial. See Appendix A.

\subsection{Procedure}

Participants were recruited consecutively through personal contact as they came for follow-up care at the clinic. Potential participants (Those that satisfy the inclusion and exclusion criteria) were informed of the nature of the study, the anticipated amount of time required for participation. Patients were informed that their participation is voluntary and that there are no penalties for non-participation. Informed consent was obtained from all participants before the administration of the instruments.

\subsection{Patient Selection}

A sample size of one hundred patients that satisfied the International Classification of Diseases Version 10 (ICD-10) for research were recruited for this study. This sample size was based on the findings of recent studies were most drug trials are carried out. It was reported among American adults that less than 10\% knew "a lot" about drug trials [8]. In developing countries this is expected to be much lower.

The ICD-10 manual contains the diagnostic criteria for various mental disorders for research purposes. Participants, documented symptoms and signs in the medical files must satisfy these criteria to be recruited for the study. Patients with active psychotic symptoms and co-morbid medical conditions were excluded from the study.

\subsection{Statistical Analysis}

Data collected was analyzed using the Statistical Package for Social Sciences (SPSS) version 20. Numerical data like age of participants was stratified and the percentage of the number of participants in each stratum calculated. For categorical variables, descriptive statistics included frequency distributions and cross tabulations in each category. The proportions were compared using chi-square $\left(\chi^{2}\right)$ test. The data was represented in tables to differentiate the demographic and response (to questions on awareness and perception) variables compared. All tests of significance were set at $\mathrm{p}<0.05$. Variables that have common associations and are statistically significant were further subjected to logistic regression analysis to determine the predictors of the awareness and perception of the drug trials questions.

\subsection{Results}

A total of 100 patients satisfied the ICD-10 Diagnostic criteria for research for various mental disorders. $56 \%$ were males while $44 \%$ were females. Most of them were married (67\%) and unemployed (67\%). They were mainly of the Hausa 
tribe with predominantly Muslims. Most of the participants are uneducated (71\%) and majority were of the age range 25 - 34 years. Full description of socio-demographic factors are in Table 1.

When participants were asked if they were aware of drug trials, about half (51\%) reported being aware and 49\% were not aware. See Figure 1.

Also, $50 \%$ of the participants were not willing to participate in a drug trial. $65 \%$ percent considered it highly risky to participate. Females were more likely to be unsure whether informed consent will be needed for drug trials $\left(\chi^{2}=7.65\right.$, $\mathrm{p}=0.02$ ). The married were more likely to be unaware of drug trials in clinical research $\left(\chi^{2}=9.30, p=0.002\right)$. The unmarried were more likely to be informed about drug trials through the media. Full details are as shown in Table 2.

Logistic regression analysis of the common associations of socio-demographic factors with some of the variables showed that being a male was a predictor of the need for school authorities consent to be sought when doing research among school children $(\mathrm{B}=1.40, \mathrm{p}=0.01)$. Also, being unmarried was a predictor of getting information about drug trials from the television $(B=1.28, p=0.02)$. Full details are shown in Table 3.

Table 1. Sociodemographic factors.

\begin{tabular}{|c|c|c|}
\hline $\mathrm{s} / \mathrm{n}$ & Variable & $\mathrm{n} / \%$ \\
\hline \multirow{3}{*}{1.} & Gender & \\
\hline & Male & $56(56 \%)$ \\
\hline & Female & $44(44 \%)$ \\
\hline \multirow{3}{*}{2.} & Marital Status & \\
\hline & Married & $67(67 \%)$ \\
\hline & Unmarried & $33(33 \%)$ \\
\hline \multirow{3}{*}{3.} & Religion & \\
\hline & Christianity & $1(1 \%)$ \\
\hline & Islam & $99(99 \%)$ \\
\hline \multirow{5}{*}{4.} & Tribe & \\
\hline & Fulani & $4(4 \%)$ \\
\hline & Hausa & $93(93 \%)$ \\
\hline & Igbo & $1(1 \%)$ \\
\hline & Yoruba & $2(2 \%)$ \\
\hline \multirow{3}{*}{5.} & Employment & \\
\hline & Employed & $33(33 \%)$ \\
\hline & Unemployed & $67(67 \%)$ \\
\hline \multirow{7}{*}{6.} & Age (years) & \\
\hline & $15-24$ & $20(20 \%)$ \\
\hline & $25-34$ & $42(42 \%)$ \\
\hline & $35-44$ & $19(19 \%)$ \\
\hline & $45-54$ & $11(11 \%)$ \\
\hline & $55-64$ & $3(3 \%)$ \\
\hline & $65-74$ & $5(5 \%)$ \\
\hline \multirow{3}{*}{7.} & Education (Formal) & \\
\hline & Educated & $29(29 \%)$ \\
\hline & Uneducated & $71(71 \%)$ \\
\hline
\end{tabular}


AWARENESS

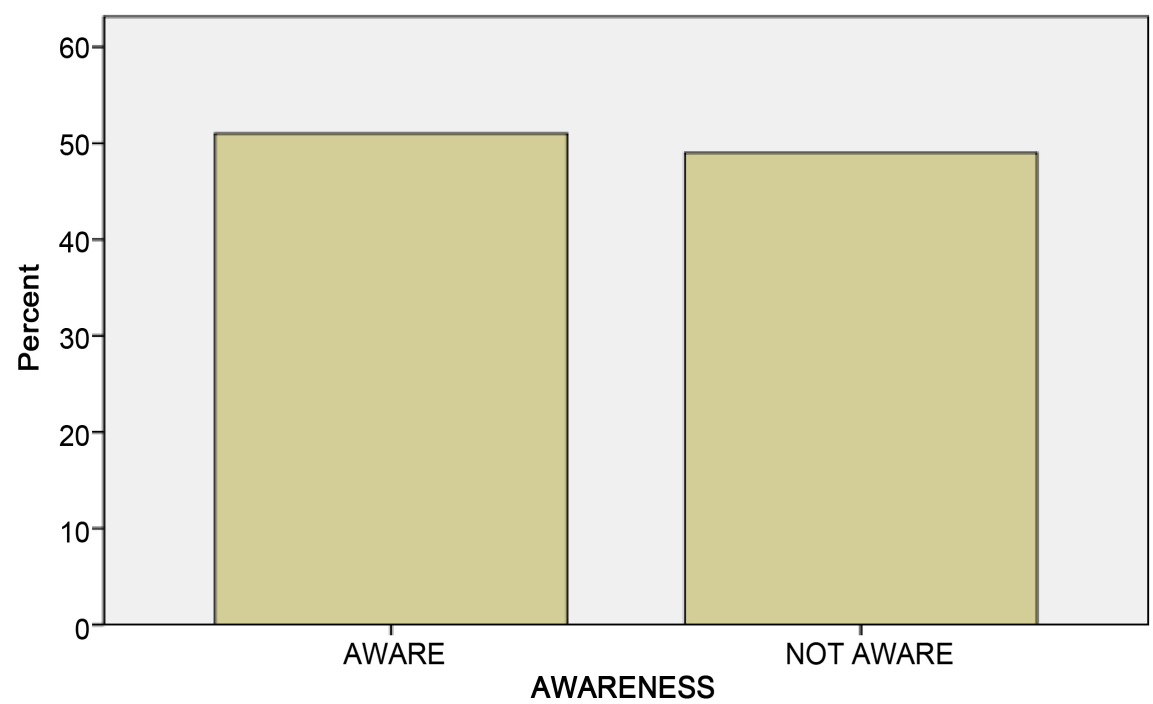

Figure 1. Awareness of drug trial patients with mental disorders.

Table 2. Association between Sociodemographic factors and Awareness/Perception Questions (see Apendix A below for the list of questions on the questionnaire). Table shows mainly associations with significant findings except for Question E5, B6, Q1, Q2, $\mathrm{B} 6$ and $\mathrm{O} 2$. Other non-significant findings are not shown.

\begin{tabular}{|c|c|c|c|}
\hline Question & Variables compared & $\chi^{2} /$ Fisher's exact & $P$ value \\
\hline B2 & No/Yes Vs. Hausa/Non-Hausa & $\chi^{2}=4.53$ & 0.03 \\
\hline $\mathrm{O} 4$ & No/Yes Vs. Hausa/non-Hausa & $\chi^{2}=11.83$ & $\mathrm{P}<0.01$ \\
\hline E5 & $\begin{array}{c}\text { No/Yes Vs. } \\
\text { organic/Non-organic-psychiatric } \\
\text { disorders }\end{array}$ & Fisher's exact $=1$ & $\mathrm{P}>0.05$ \\
\hline B6 & No/Yes. Vs. Christianity/Islam & Fisher's exact $=0.17$ & $\mathrm{P}>0.05$ \\
\hline Q1 & No/Yes. Vs. Christianity/Islam & Fisher's exact $=0.16$ & $\mathrm{P}>0.05$ \\
\hline Q2 & No/Yes. Vs. Christianity/Islam & Fisher's exact $=0.16$ & $\mathrm{P}>0.05$ \\
\hline B6 & No/Yes. Vs. 25 - 34 yrs/Other age groups & $\chi^{2}=0.38$ & 0.54 \\
\hline $\mathrm{O} 2$ & No/Yes. Vs. 25 - 34 yrs/Other age groups & Fisher's exact $=0.26$ & $\mathrm{P}>0.05$ \\
\hline E1 & No/Yes. Vs. 25 - 34 yrs/Other age groups & $\chi^{2}=5.78$ & 0.02 \\
\hline E3 & No/Yes. Vs. 25 - 34 yrs/Other age groups & $\chi^{2}=5.43$ & 0.02 \\
\hline $\mathrm{F}$ & Don't-know/No/Yes. Vs. Female/Male & $\chi^{2}=7.65$ & 0.02 \\
\hline A & $\begin{array}{c}\text { Don't-know/No/Yes. Vs. } \\
\text { Married/unmarried }\end{array}$ & $\chi^{2}=10.23$ & 0.01 \\
\hline
\end{tabular}


Table 3. Logistic regression analysis of variables with common awareness/perception association.

Question N3

\begin{tabular}{cccccccc}
\hline Variable & B & Wald & df & P value & Exp (B) & $95 \%$ CI (Lower) & 95\% CI (Upper) \\
\hline $\begin{array}{c}\text { Marital } \\
\text { Status (1) }\end{array}$ & 1.55 & 3.72 & 1 & 0.05 & 4.72 & 0.98 & 22.83 \\
Gender (1) & 1.40 & 6.40 & 1 & 0.01 & 4.04 & 1.37 & 11.93 \\
\hline
\end{tabular}

Question N2

\begin{tabular}{cccccccc}
\hline Variable & B & Wald & df & P value & $\operatorname{Exp}(B)$ & $95 \%$ CI (Lower) & 95\% CI (Upper) \\
\hline $\begin{array}{c}\text { Marital } \\
\text { Status (1) }\end{array}$ & 1.41 & 3.05 & 1 & 0.08 & 4.10 & 0.84 & 20.00 \\
Gender (1) & 1.11 & 3.94 & 1 & 0.05 & 3.05 & 1.02 & 9.15 \\
\hline
\end{tabular}

Question B1

\begin{tabular}{cccccccc}
\hline Variable & B & Wald & df & P value & Exp (B) & 95\% CI (Lower) & 95\% CI (Upper) \\
\hline $\begin{array}{c}\text { Marital } \\
\text { Status (1) }\end{array}$ & 1.28 & 5.77 & 1 & 0.02 & 3.59 & 1.27 & 10.16 \\
Gender (1) & 0.88 & 2.17 & 1 & 0.14 & 2.40 & 0.75 & 7.69 \\
\hline
\end{tabular}

\begin{tabular}{cccccccc}
\hline \multicolumn{7}{c}{ Question B6 } \\
\hline Variable & B & Wald & df & P value & Exp (B) & $95 \%$ CI (Lower) & 95\% CI (Upper) \\
\hline $\begin{array}{c}\text { Marital } \\
\text { Status (1) }\end{array}$ & 0.88 & 1.58 & 1 & 0.21 & 2.41 & 0.61 & 9.49 \\
$\begin{array}{c}\text { Religion (1) } \\
\text {-21.62 }\end{array}$ & 0.00 & 1 & 1.00 & 0.00 & 0.00 & \\
$\begin{array}{c}\text { Age group } \\
(1)\end{array}$ & -0.85 & 0.71 & 1 & 0.23 & 0.43 & 0.11 & 1.73 \\
\hline
\end{tabular}

\begin{tabular}{|c|c|c|c|c|c|c|c|}
\hline \multicolumn{8}{|c|}{ Question M } \\
\hline Variable & B & Wald & $\mathrm{df}$ & $\mathrm{P}$ value & $\operatorname{Exp}(B)$ & 95\% CI (Lower) & 95\% CI (Upper) \\
\hline Gender (1) & 0.50 & 0.44 & 1 & 0.51 & 1.66 & 0.37 & 7.33 \\
\hline $\begin{array}{c}\text { Marital } \\
\text { Status (1) }\end{array}$ & 19.16 & 0.00 & 1 & 1.00 & 2.09 & 0.00 & \\
\hline Tribe (1) & -19.40 & 0.00 & 1 & 1.00 & 0.00 & 0.00 & \\
\hline \multicolumn{8}{|c|}{ Question $\mathrm{H}$} \\
\hline Variable & B & Wald & $\mathrm{df}$ & $P$ value & $\operatorname{Exp}(B)$ & 95\% CI (Lower) & 95\% CI (Upper) \\
\hline Gender (1) & 0.59 & 1.75 & 1 & 0.19 & 1.80 & 0.75 & 4.33 \\
\hline $\begin{array}{c}\text { Marital } \\
\text { status (1) }\end{array}$ & 0.77 & 2.35 & 1 & 0.13 & 2.16 & 0.81 & 5.80 \\
\hline
\end{tabular}

\{NOTE: Maritalstatus (1)-Unmarried, Gender (1)-Male, Religion (1)-Islam, Age group (1)-(25 - 34 years), Tribe (1)-Hausa\}.

\section{Discussion}

The main thrust of this study is on the assessment of awareness and perception of drug trial among patients with mental disorders. Majority of the participants 
were young adults which implies that the decision to participate in a drug trial will depend a lot on the individuals themselves. This is because participants in the extremes of life (children and elderly) require inputs from significant family relatives which are usually overriding. Inability to properly engage these young adults may actually hinder the implementation of the protocol despite the willingness of relatives.

Majority were also unemployed; this can make these individuals vulnerable to coercion or abuse in drug trials. About half of the participants were aware of drug trials. This finding probably reflects a general knowledge of drug trials but not a depth of knowledge sufficient for participation in such research activities. This finding is lower than that reported by studies done in western countries like America were $74 \%$ and $80 \%$ were reported in two studies respectively [9] [21]. However, the unwillingness of half of the participants to participate in drug trials portends a not very good outlook among these subjects in drug trials research. There are mixed findings in other challenging disease conditions like cancer where about $69 \%$ have been reported to be willing to participate in drug trials [22] and over $80 \%$ unwilling to participates [23]. Furthermore, the greater percentage of participants' perception of drug trials as highly risky will likely also negatively affect participation. A study conducted in about 68 countries reported that $90 \%$ believed drug trials are generally safe [24], while another study among American adults reported that $43 \%$ considered it too risky [9].

The finding of the married being more likely to be unaware of drug trials may be due to majority of the participants being married which might have influenced the finding of the association of married participants with unawareness of drug trials. This may also require further investigation into other possible reasons for this finding. The unmarried were however more likely predictors of awareness through the television. This is in keeping with the increased use of media platforms and internet in the last decade especially among young adults in this country. It is possible that they have heard more about the litigations of drug companies due to failed drug trails and complications from the media and internet. One study found increased use of the internet as a predictor of drug trial awareness [21]. Little is known through the media about its importance and benefits. This might also have influenced the greater number of participants considering drug trials as highly risky. This impression is also seen among young adults in productive age group (24 - 34 years) higher preference for the use of the elderly in drug trials. This may likely be due to the assumption that risky research activities should be done among people that a closer to death than among young people. Those below 24 years and above 34 years were more likely to recommend healthy individuals for drug trials than the young adults. It is possible that older people and the younger age group (below 24 years) consider the 24 - 34 years age group as the healthy ones.

The finding of females' association with being unsure of whether their consent will be needed may likely reflect an underlying social-cultural pattern as pre- 
viously described [25]. This socio-cultural pattern creates a need to be holistic during the consenting process in drug trials. This is because socio-cultural factors play pivotal role in influencing the decisions of participants. In typical Northern Nigeria the consent of married females is usually determined by their husbands' permission to participate in such research activities despite its necessity or urgency. Designs of drug trials in such socio-cultural setting will therefore require proper engagement with their husbands even before married female participants are approached for participation.

High socio-economic problems have been associated with these patients in previous studies [26] [27] this can be linked to the high rate of unemployment and lack of formal education of majority of the participants in this study. This makes participants more vulnerable to inducements and coercion in drug trials.

The participants of the minority tribe (non-Hausa) were more likely to be aware of drug trials from reading newspapers and to request for other forms compensation in instances of adverse drug reactions than the predominant tribe (Hausa) during drug trials. These findings may be due to the rather small number of the minority tribe rather than inherent attributes. However further examination of these findings in future studies may be necessary.

\section{Conclusion}

The level of awareness of drug trials among psychiatric patients appears to be poor and majority of them consider it a highly risky research to participate in. Socio-economic problems encountered by participants with psychiatric disorders need to be considered in the design of drug trials among them. The female patients are likely to be more vulnerable to coercion and abuse in such research activities.

\section{Recommendations}

There should be concerted effort to correct the wrong perception of drug trials through the use of media platforms and public enlightenment programmes.

The design of drug trials among psychiatric patients should adequately include a legal framework that takes into cognizance their vulnerability and how to ensure that the consent process is not compromised. This will require a multidisciplinary team of experts in the field of Mental Health who will provide guidance on the development of a protocol that shows an understanding of the disorder and its psychopathology.

Researchers should consider the addition of long-term benefits in the research design to participants that will be supportive, considering the continuous social challenges they face due to mental disorders. This will go a long way in sustaining the quality of life of participants beyond the research period and encourage participation.

\section{Limitations}

It was a cross-section study and may not be sensitive to changes in awareness 
and perception over a period of time.

It was a hospital based study, which may not reflect what obtains in the community.

\section{Declaration}

All authors agree with the content of the manuscript and there are no conflicts of interest between/among the authors.

\section{References}

[1] WHO (2018) Mental Disorders. WHO Fact Sheets. http://www.who.int/en/news-room/fact-sheets/detail/mental-disorders

[2] Croft, H. (2017) Mental Health Clinical Trials. https://www.healthyplace.com/other-info/clinical-trials/mental-health-clinical-trials

[3] Nierenberg, A. (2007) The Contribution of Clinical Trials in Psychiatry: Beyond Basic Science. Revista de Psiquiatria do Rio Grande do Sul, 29, 13-14.

http://www.scielo.br/scielo.php?script=sci_arttext\&pid=S0101-81082007000100005 \&lng=pt\&tlng=pt

[4] Meinert, C.L. and Tonascia, S. (1986) Clinical Trials: Design, Conduct, and Analysis. Oxford University Press, Oxford.

https://books.google.com/?id=i1oAxuE29MUC\&pg=PA3\&lpg=PA3\&q https://doi.org/10.1093/acprof:oso/9780195035681.001.0001

[5] Trouiller, P., Olliaro, P., Torreele, E., Orbinski, J., Laing, R. and Ford, N. (2002) Drug Development for Neglected Diseases: A Deficient Market and a Public-Health Policy Failure. The Lancet, 359, 2188-2194.

https://www.sciencedirect.com/science/article/pii/S0140673602090967

[6] WHO (2001) Mental Health Determinants and Populations Department of Mental Health and Substance Dependence. Geneva.

http://apps.who.int/iris/bitstream/handle/10665/67160/WHO_NMH_MSD_MDP_0 1.3_P1.pdf?sequence $=1$ \&isAllowed $=\mathrm{y}$

[7] WHO (2015) Clinical Trials. http://www.who.int/topics/clinical_trials/en

[8] Miller, M. (2017) Clinical Trials Awareness Week. http://www.imarcresearch.com

[9] New National Public Opinion Survey Research America. http://www.researchamerica.org

[10] NIMH (2015) A Participant's Guide to Mental Health Clinical Research. Bethesda, Report No. 310-443-4513.

https://catalyst.harvard.edu/pdf/regulatory/nimh-participants-guide.pdf

[11] Phase 0 Trials: A Platform for Drug Development? The Lancet, 374, 176. http://www.ncbi.nlm.nih.gov/pubmed/19616703

[12] Gad, S.C. (2009) Clinical Trials Handbook. Wiley, Hoboken, 1225 p. https://books.google.com/books?id=d8GxG0d9rpgC\&pg=PA118

[13] Brintnall-Karabelas, J., Sung, S., Cadman, M.E., Squires, C., Whorton, K. and Pao, M. (2011) Improving Recruitment in Clinical Trials: Why Eligible Participants Decline. Journal of Empirical Research on Human Research Ethics, 6, 69-74. http://www.ncbi.nlm.nih.gov/pubmed/21460590

[14] Van Spall, H.G.C., Toren, A., Kiss, A. and Fowler, R.A. (2007) Eligibility Criteria of Randomized Controlled Trials Published in High-Impact General Medical Journals: A Systematic Sampling Review. JAMA, 297, 1233-1240. 
http://www.ncbi.nlm.nih.gov/pubmed/17374817

[15] ICH (2008) ICH Official Web Site: ICH. ich.org. http://www.ich.org

[16] Kharawala, S. and Dalal, J. (2011) Challenges in Conducting Psychiatry Studies in India. Perspectives in Clinical Research, 2, 8-12. http://www.ncbi.nlm.nih.gov/pubmed/21584176

[17] Hannan, E.L. (2008) Randomized Clinical Trials and Observational Studies: Guidelines for Assessing Respective Strengths and Limitations. JACC: Cardiovascular Interventions, 1, 211-217. http://www.ncbi.nlm.nih.gov/pubmed/19463302

[18] Llewellyn-Thomas, H.A., McGreal, M.J., Thiel, E.C., Fine, S. and Erlichman, C. (1991) Patients' Willingness to Enter Clinical Trials: Measuring the Association with Perceived Benefit and Preference for Decision Participation. Social Science \& Medicine, 32, 35-42. http://www.ncbi.nlm.nih.gov/pubmed/2008619

[19] March, J.S., Silva, S.G., Compton, S., Shapiro, M., Califf, R., March, J.S., Silva, S.G., Compton, S., Shapiro, M., Califf, R. and Krishnan, R. (2005) The Case for Practical Clinical Trials in Psychiatry. American Journal of Psychiatry, 162, 836-846.

https://doi.org/10.1176/appi.ajp.162.5.836

[20] Tansella, M., Thornicroft, G., Barbui, C., Cipriani, A. and Saraceno, B. (2006) Seven Criteria for Improving Effectiveness Trials in Psychiatry. Psychological Medicine, 36, 711. http://www.ncbi.nlm.nih.gov/pubmed/16512969

[21] Leiter, A., Diefenbach, M.A., Doucette, J., Oh, W.K. and Galsky, M.D. (2015) Clinical Trials Awareness: Changes over Time and Sociodemographic Disparities. Clinical Trials, 12, 215-223. https://doi.org/10.1177/1740774515571917

[22] Fenton, L., Rigney, M. and Herbst, R.S. (2009) Clinical Trial Awareness, Attitude and Participation among Patients with Cancer and Oncologists. Community Oncology, 6, 207-228. https://doi.org/10.1016/S1548-5315(11)70546-0

[23] Dias, A.L., Chao, J.H., Lee, D., Wu, Y. and Kloecker, G.H. (2016) Patient's Perception Concerning Clinical Trials in Oncology Patients. Contemporary Clinical Trials Communication, 4, 179-185. https://doi.org/10.1016/j.conctc.2016.09.005

[24] Anderson, A., Borfitz, D. and Getz, K. (2017) Global Public Attitudes about Clinical Research and Patient Experiences with Clinical Trials. JAMA Network Open, 1, e182969.

[25] United Nations University Website. http://unu.edu

[26] Molarius, A., et al. (2009) Mental Health Symptoms in Relation to Socio-Economic Conditions and Lifestyle Factors-A Population Based Study in Sweden. BMC Public Health, 9, 302. https://doi.org/10.1186/1471-2458-9-302

[27] Taylor, R., Page, A., Morrell, S. and Carter, G. (2004) Socio-Ecnomic Differentials in Mental Disorders and Suicide Attempts in Australia. British Journal of Psychiatry, 185, 486-493. https://doi.org/10.1192/bjp.185.6.486 


\section{Appendix A}

Proforma

Questions

A. Are you aware that new drugs are being tested before they are released into the market?

B. If you are aware where did you get your information from?

Television/Radio-B1, Newspaper-B2, Friends/Relatives-B3, Internet-B4, Doctors-B5, Others-B6.

C. Do you know that drugs are first tested in animals?

D. Do you know that after the animal testing it is then tested on humans?

E. If yes, tick the appropriate group of persons that should be used for the test.

Healthy individuals-E1, critically ill patients-E2, Elderly patients-E3, mentally ill patients-E4, others specify-E5.

F. Is the participant's consent required before carrying out the test?

G. Should the test be carried out on participants for free?

H. Should the new drug be tested and compared with other drugs that are used to treat the same disease?

I. How will you feel if you are asked to participate in the testing of a new drug?

J. Can you willingly offer yourself to participate in testing a new drug?

K. If yes, why?

No other medicine available to treat the disease-K1, If the drug is given free of Charge-K2, If I have used the new drug before-K3, I trust the Doctors-K4, If there is hope of cure with the new drug-K5,others specify-K6.

L. How will you feel not knowing the drug you are being given during the drug test?

M. How risky do you think it is participating in the test of a new drug?

Dangerous-M1, High risk-M2, Moderate risk-M3, Low risk-M4, Safe-M5.

$\mathrm{N}$. Who should consent to the testing of a new drug in children?

Parent-N1, Child-N2, School Authourities-N3, Others specify-N4.

O. In case of adverse effects during the drug test, what should be done to the participants affected?

Stoppage of the drug-O1, Prompt medical attention-O2, Compensation-O3, Others specify-O4.

P. Do you think you need to be paid before participating in the testing of a new drug?

Q. If yes,

Give reason(s) why-Q1.

How much do you think you should be paid? -Q2. 